\title{
Texture Features and Segmentation Based on Multifractal Approach
}

\author{
Mohamed Abadi and Enguerran Grandchamp \\ GRIMAAG UAG, Campus de Fouillole, French West Indies University, \\ 97157 Pointe-à-Pitre Cedex Guadeloupe France \\ \{mabadi, egrandch\} @univ-ag.fr
}

\begin{abstract}
In this paper, we use a multifractal approach based on the computation of two spectrums for image analysis and texture segmentation problems. The two spectrums are the Legendre Spectrum, determined by classical methods, and the Large Deviation Spectrum, determined by kernel density estimation. We propose a way for the fusion of these two spectrums to improve textured image segmentation results. An unsupervised k-means is used as clustering approach for the texture classification. The algorithm is applied on mosaic image built using IKONOS images and various natural textures from the Brodatz album. The segmentation obtained with our approach gives better results than the application of each spectrum separately.
\end{abstract}

Keywords: Multifractal theory, multifractal spectrum, wavelets, texture segmentation, high and very high spatial resolution image.

\section{Introduction}

This work has been conducted within the CESAR project (Arborescent species classification). The main objective is to reinforce monitoring measurements and to improve durable management of forest resources for ecological considerations. The first step is to obtain a forest identification using very high satellite images such as QUICKBIRD or IKONOS images.

Since we consider that forests could be identified using their texture features our approach is to segment the images into homogeneous textured regions. Indeed, natural images are composed of different and various textures often considered as an infinite combination. With the apparition of very high resolution satellite images, small structures are now textured instead of having a homogeneous mean intensity.

Texture analysis has been studied for a long time using different approaches. A lot of methods are directly based upon the intensity of an image. These include grey-level co-occurrence matrices (GLCM) [1], [2], autocorrelation function analysis [1], moment based texture segmentation [3], two-dimensional filtering in the spatial and frequency domain [4], [5], and the fractal analysis [6].

In order to perform the segmentation we have to characterize each texture. To achieve this goal we have used the multifractal tool based on the Legendre and Large Deviation Spectrum (LS and LDS) [7]. Both spectrums exploit the local and global 
regularity of the image using the distribution of singularity exponent [8] (Hölder coefficient or Lipschitz exponent). We justify our choice by the fact that studied images are taken from natural scenes having non-stationary characteristics resulting in complex structures (multifractal) [9]. The analysis of the singular structures of the image [10] enables as to establish a relation between a geometrical description and a statistical description for local and global grey-levels.

These two spectrums are two different ways to compute the same parameter. LS is a statistical approach and LDS a geometrical approach [7].

The article is composed as follow: section 2 explains different approaches used in literature for texture segmentation. Section 3 defines the multifractal approach and the spectrum computation in a two-dimensional case. Section 4 describes the algorithm we use to compute the texture features. Experiments and results are given in Section 5. Section 6 gives some concluding remarks.

\section{Texture Segmentation Approach}

In the literature there are two important aspects of texture image segmentation procedures, namely feature extraction and classification. There are several methods allowing to extract texture features of images that we can regroup in four large topics: statistical methods (co-occurrence matrices, autocorrelation features [1], [2] moment features [11]), geometrical methods (Voronoi tessellation features [11], structural methods [12]), model based methods (random field models [13], [14], fractals [15], [16]), and signal processing methods (spatial domain filters [17], Fourier domain filtering [18], Gabor and wavelet models [5]). Classification methods are grouped in two categories: supervised and unsupervised classification methods (maximum likelihood classifier [19], Bayesian classification [20], neural networks [21], ...).

In the scope of this article we have chosen a multifractal approach and an unsupervised k-means classification.

\section{Multifractal Approach}

The multifractal formalism is a nice tool for characterizing and describing the statistical and geometrical properties of images from real world scenes [10]. The main idea in this approach is to built a positive measure $\mu$ on the image $I(\vec{x})$, where $\vec{x} \in \Re^{2}$ represents the coordinate vector. Treatments will be made using the measure instead of the image. For each set $A$, the measure $\mu(A)$ is given by:

$$
\mu(A)=\int_{A} I(\vec{x}) d \vec{x}
$$

hence $\mu$ is compactly supported and thus finite.

We quickly present in the following the computation of Legendre and Large Deviation Spectrum. 


\subsection{Legendre Spectrum (LS)}

We define here $\mu$ as a Borel probability measure over $[0,1] \times[0,1]$. Let $v_{n}$ be an increasing sequence of positive integers, and let us define:

$$
I_{i, j, n}=\left[\frac{i}{v_{n}}, \frac{i+1}{v_{n}}\right] \times\left[\frac{j}{v_{n}}, \frac{j+1}{v_{n}}\right]
$$

We directly compute some kind of "moments" or generalized dimensions of our measure. To perform this calculation, we will use the Differential Box Counting (DBC) method defined by Chaudhuri and Sarker (1992) [22]. The generalized dimension of order $q, q \in \mathfrak{R}$, is defined by :

$$
\mu\left[I_{n}(i, j)\right]^{q}=\frac{\left[P_{n}(i, j)\right]^{q}}{\sum_{i} \sum_{j}\left[P_{n}(i, j)\right]^{q}}
$$

Where $P_{n}(i, j)$ is the probability estimation in a ball of radius $v_{n}$.

Then the multifractal spectrum is defined as follows:

$$
f(q)=\lim _{n \rightarrow \infty} \frac{\sum_{i} \sum_{j} \mu\left[I_{n}(i, j)\right]^{q} * \log \left\{\mu\left[I_{n}(i, j)\right]^{q}\right\}}{\log \left(v_{n}\right)}
$$

and the singularity exponent as

$$
\alpha(q)=\lim _{n \rightarrow \infty} \frac{\sum_{i} \sum_{j} \mu\left[I_{n}(i, j)\right]^{q} * \log \left[P_{n}(i, j)\right]}{\log \left(v_{n}\right)}
$$

\subsection{Large Deviation Spectrum (LDS)}

Let us consider the projections of the measure $\mu$ over an appropriate wavelet $\psi$ around a point $\vec{x}_{0}$ who allows evaluating $\mu$ over balls $B$ centred on $\vec{x}_{0}$ with different radius $r=\frac{1}{v_{n}}$

$$
T_{\psi} \mu\left(\vec{x}_{0}, r\right) \equiv \frac{1}{r^{2}} \int_{B} \mu(\vec{x}) \psi\left(\frac{\vec{x}_{0}-\vec{x}}{r}\right)
$$

The wavelet projection provides the same singularity exponents as $\mu$ ([9]), that is: 


$$
T_{\psi} \mu\left(\vec{x}_{0}, r\right) \approx r^{\alpha\left(\vec{x}_{0}\right)}
$$

It has been established in [9] that natural images can be modelled by this equation.

Starting from this equation we can estimate the singularity exponents as being the slope of the linear regression in the following way:

$$
\alpha\left(\vec{x}_{0}\right)=\lim _{r \rightarrow 0} \frac{\log \left[T_{\psi} \mu\left(\vec{x}_{0}, r\right)\right]}{\log (r)}
$$

After the computation of the singularity exponent at each point by using the above equation, the LDS is estimated [7] by

$$
f_{g}(h)=\lim _{\varepsilon \rightarrow 0} \lim _{r \rightarrow \infty} \frac{\log \left[N_{r}^{\varepsilon}(h)\right]}{\log (r)}
$$

where $N_{r}^{\varepsilon}(h)=\#\{\alpha / h \in[\alpha-\varepsilon, \alpha+\varepsilon]\} \approx \varepsilon K_{\varepsilon} * p_{r}(h), *$ is the convolution product, $p_{r}(h)$ the density of $\alpha(\vec{x})$ and $K_{\varepsilon}=\frac{1}{\varepsilon} K\left(\frac{h}{\varepsilon}\right)$ a rectangular kernel [19]

$$
K(t)= \begin{cases}1 & \text { if } \quad t \in\left[-\frac{1}{2}, \frac{1}{2}\right] \\ 0 & \text { outside }\end{cases}
$$

\section{Proposed Algorithm}

In this section, we expose the method which will allow us to fuse the two previous spectrums (LS and LDS) in order to obtain a single value for each pixel $(i, j)$. This value will be denoted $F(i, j)$ and explained in the following as well as the resulting algorithm.

\subsection{EPNSQ Filtering Approach}

The Edge Preserving Noise Smoothing Quadrant (EPNSQ) filtering approach was introduced by [23] with the objective of estimating the local statistics and to reduce the misclassification inside texture regions.

This method could be applied on any feature computed from the original image. It has often been applied on fractal dimension estimations and on laws filter, etc. but never on LS and LDS. The EPNSQ can be applied on these two spectrums given the fact that there are two different approaches (geometrical and statistical) to compute the same parameter.

The EPNSQ algorithm performs as follow.

Let us consider $\mathrm{q}$ features computed from the image. A sliding window of size $(2 w+1) \times(2 w+1)$ centered on each coordinate $(i, j)$ will be used for each 
feature. This window is split into four quadrants. Fig. 1. illustrates this with $w=2$ and $q=1$, thus the size of each $W_{k}$ is $(w+1) \times(w+1) \equiv 3 \times 3$.

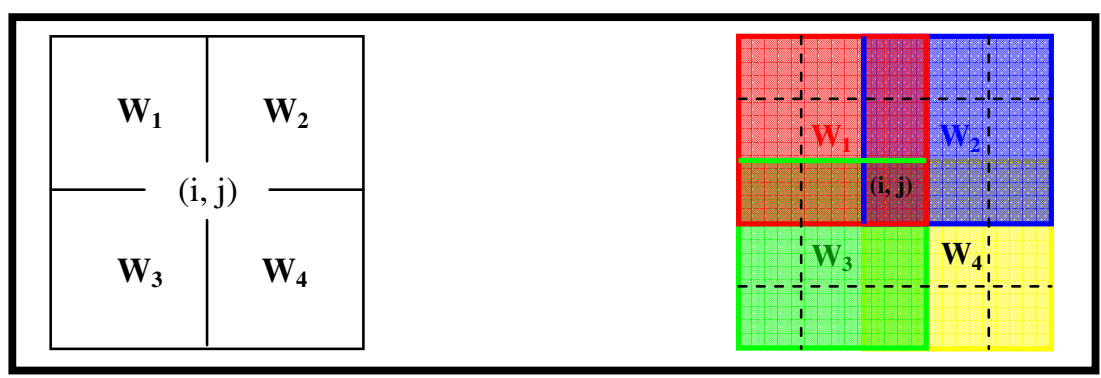

Fig. 1. Four quadrants and an example for a $w=2$

For each quadrant $k$ and each feature $p$ we compute the local variance $V_{W_{k}^{p}}$. We select the quadrant $k^{*}$ and the feature $p^{*}$ with the minimal variance

$$
V_{W_{k^{*}}^{p^{*}}}=\min \left(V_{W_{k}^{p}}\right) \quad k=1, \cdots, 4 \quad p=1, \cdots, q
$$

The feature value $F(i, j)$ is the average value computed on the $W_{k^{*}}^{p^{*}}$ quadrant

$$
F(i, j)=\frac{1}{(w+1)^{2}} \sum_{n=1}^{w+1} \sum_{m=1}^{w+1} W_{k^{*}}^{p^{*}}(m, n)
$$

This value will be considered as being the most representative spectrum value for each pixel.

\subsection{Our Algorithm}

The block diagram of our algorithm is described in the following figure and consists in the following steps:

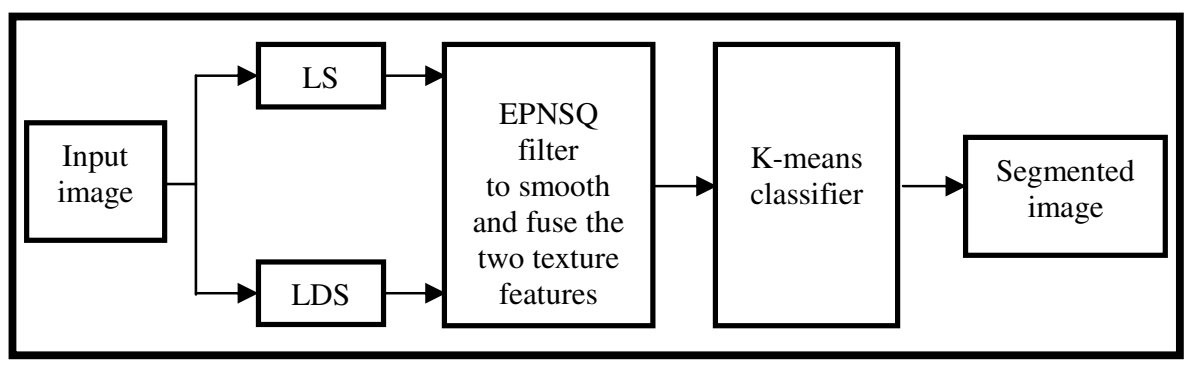

Fig. 2. Block diagram of our algorithm 
If the two spectrums (LS and LDS) are computed over the whole image, then each pixel $(i, j)$ is associated with two features and the EPNSQ filtering approach is used to compute $F(i, j)$. We perform the texture segmentation by applying a general kmeans clustering algorithm [24] on $F(i, j)$.

\section{Experimental Results}

This approach, texture features extraction and segmentation, has been tested on different mosaic images built using Brodatz album textures (D9, D57 and D92) (fig. 3) and textures extracted from IKONOS images (fig. 4). Brodatz textures have been chosen because they are close to forest textures extracted from satellite images.

The size of each mosaic image is $256 \times 256$ pixels. The texture feature $F(i, j)$ is computed using a sliding window of size $17 \times 17$. The number of classes for the $\mathrm{k}$ mean classification algorithm is set to the number of textures ( 3 in fig. 3 and 4 in fig. 4). For each figure, the k-means classification algorithm was applied on LS (b), LDS (c) and $F(i, j)(\mathrm{d})$.
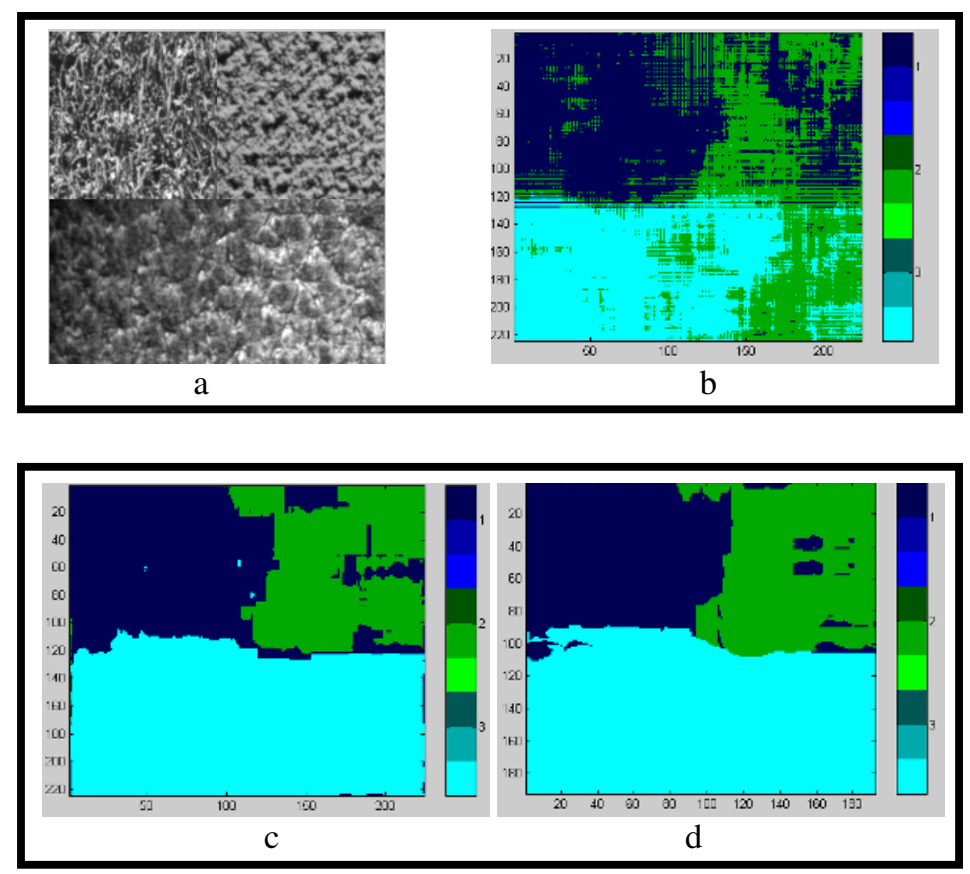

Fig. 3. a: image created from three Brodatz textures (D9, D57, D92). b: segmentation obtained using LS. c: segmentation obtained using LDS. d: segmentation obtained using our algorithm. 


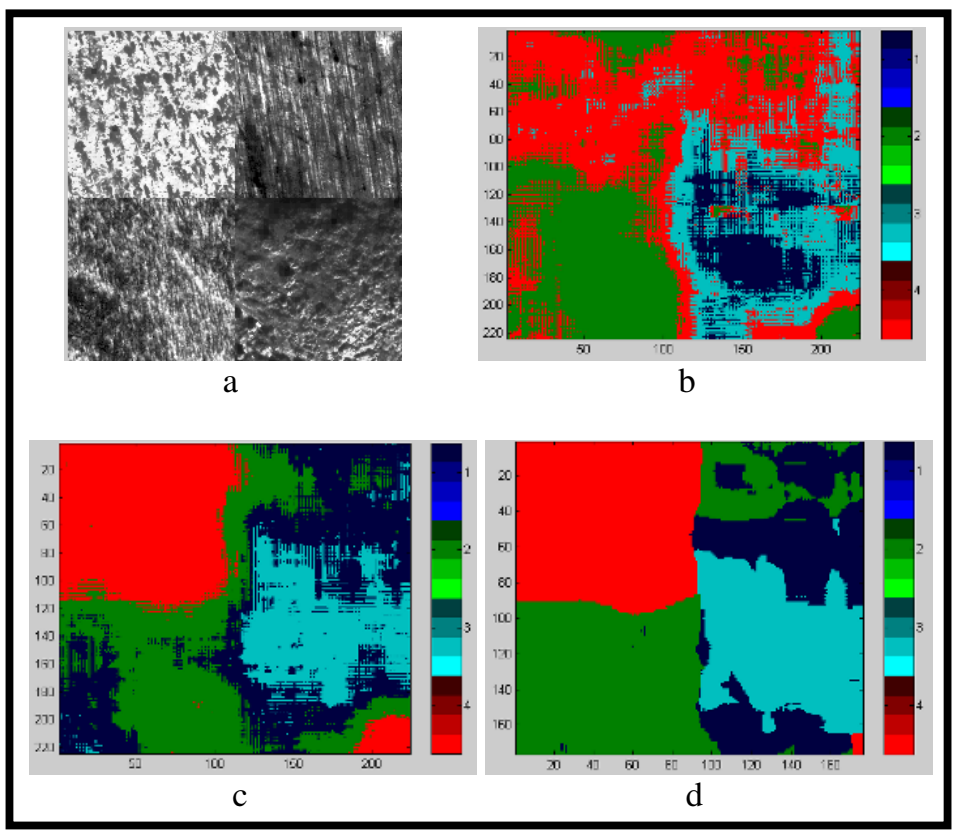

Fig. 4. a: image created from four textures extracted from IKONOS images. b: segmentation obtained using LS. c: segmentation obtained using LDS. d: segmentation obtained using our algorithm.

The results show that the classification algorithm applied on texture features $F(i, j)$ gives a better segmentation on the two kinds of images than the segmentation applied on both spectrums separately. (b) and (c) of fig. 3 and fig. 4 shows that the segmentation based in LDS is better that LS. The main contribution is that spectrum fusion improves the homogeneity of the regions.

\section{Conclusion}

In this paper we have developed a texture feature segmentation algorithm based on the multifractal spectrums. This approach first computes the Legendre and Large Deviation spectrum within localized regions of the image around each pixel. Then it computes the features $F(i, j)$ for each pixel using the EPNSQ filtering approach. Finally it classifies these features using a k-means clustering algorithm.

We note that the segmentation applied on $F(i, j)$ gives more homogeneous and compact regions. The classification error using $F(i, j)$ is lower than the error using LS or LDS.

In future works we will try to exploit the LDS using different kernel density estimation methods and we will integrate colour information. 


\section{Acknowledgements}

The authors are thankful to the European institutions for the financing of the CESAR project (Arborescent species classification) and Guadeloupe, Martinique and French Guyana areas within the "INTERREG IIIb Caribbean Space" European program.

\section{References}

1. Haralick, R.M.: Statistical and structural approaches to texture. Proceedings of the IEEE (1979) 786-804

2. Haralick, R.M., Shanmugam, K. Dinstein, I.: Textural features for image classification. IEEE Transactions on systems, Man and Cybernetics, (1973) 610-621

3. Tuceryan, M.: Moment based texture segmentation. Pattern Recognition Letters, (1994) 695-668

4. Clark,M., Bovik, A.C.: Texture segmentation using Gabor modulation/demodulation. Pattern Recognition Letters, (1987) 261-267

5. Turner, M.R.: Texture discrimination by Gabor functions. Biological Cybernetics, (1986) 71-82

6. Kaplan, L.M.: Extended Fractal Analysis for Texture Classification and Segmentation. IEEE Transaction on Image Processing, (1999) 1572-1585

7. Levy Vehel, J. : Introduction to the multifractal analysis of images, INRIA

8. Sapiro, G., Tannenbaum, A. : on invariant curve evolution and image analysis. Indiana University Mathematics Journal, (1993) 985-1010

9. Ruderman, D.: The statistics of naturel images. Network 5, (1994) 517-548

10. Grazzini, J.: Analyses multiéchelle et multifractale d'image météorologique Application à la detection de zonnes précipitantes. (2003)

11. Tuceryan, M.: Texture Analysis. In the Handbook of Pattern Recognition and Computer Vision, L.F. Pau and P.S.P. Wang(eds), World Scientific Publishing Co., (1993)

12. Julesz, B.: Textons, the Elements of Texture Perception, and Their Interactions. Nature, (1981) 91-97

13. Chatterjee, S. and Chellappa, R.: Maximum likelihood texture segmentation using Gaussian Markov random field models. P roc. IEEE Coqf Computer Vision, Graph, Pattern Recog., (1985)

14. Derin, H. and Elliot, H.: Modelling and segmentation of noisy and textured images using Gibbs random fields. IEEE Trans on Pattern Anal. and Machine. Intell, (1987) 39-55

15. Keller, J., Crownover, R. and Chen, S. :Texture Description and Segmentation through Fractal Geometry. Computer Vision Graphics and Image Processing, (1989) 150-1 60

16. Chaudhuri, B. B., Sarkar, N. and Kundu, P.: An Improved Fractal Geometry Based Texture Segmentation Technique. Proc. IEE-part E

17. Laws, K. I., Textured Image Segmentation. Ph.D. thesis, University of Southern California, (1980)

18. Campbell, F.W. and Robson, J.G.: Application of Fourier Analysis to the Visibility of Gratings. Journal of Physiology, (1968) 551-566

19. O. Duda, R., E. Hart, P. : Pattern classification and scene analysis. Wiley-Iterscience, New York, (1973).

20. Cheeseman, P., Self, M., Kelly, J., Stutz, J., Taylor, W., Freeman., D. : Bayesian classification. In Seventh National Conference on Artificial Intelligence, (1988) 607-611 
21. Grayson, M. : The heat equation shrinks embedded plane curves to round points. J. Differential Geometry, vol. 26, (1987) 285-314

22. Chaudhuri, B.B., Sarker, N.: An Efficient Approach to Estimate Fractal Dimensions of Textural Images, Pattern recognition, (1992) 1035-1041

23. Donnay, J.-P., Barnsley, M.J., Longley, P.A. (eds.) : Remote Sensing and Urban Analysis, GISDATA 9, Taylor \& Francis, London, (2000) 3-18

24. Tou, J.T., Gonzalez, R.C.: Pattern Recognition Principles, Addison-Wesley, Reading, MA, (1982) 